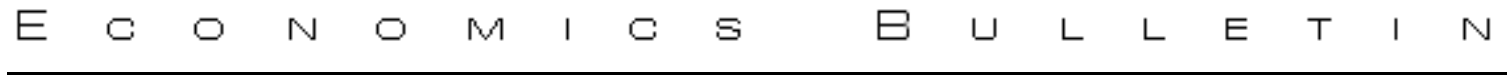

\title{
Common Components in Firms' Growth and the Sectors Scaling Puzzle
}

\author{
Antonio Palestrini \\ University of Teramo
}

\begin{abstract}
In this paper we show how a simple modification of a well-known multiplicative process of firms' growth, taking into account common and idiosyncratic elements, allows to reconcile an old aggregate-sector puzzle (Quandt, 1966) on firms' size distribution reported in the literature.
\end{abstract}

Citation: Palestrini, Antonio, (2008) "Common Components in Firms' Growth and the Sectors Scaling Puzzle." Economics Bulletin, Vol. 12, No. 34 pp. 1-8

Submitted: September 8, 2008. Accepted: December 13, 2008.

URL: http://economicsbulletin.vanderbilt.edu/2008/volume12/EB-08L00008A.pdf 


\title{
Common Components in Firms' Growth and the Sectors Scaling Puzzle
}

\author{
R. Axtell \\ The Brookings Institution \\ M. Gallegati \\ Università Politecnica delle Marche \\ A. Palestrini \\ University of Teramo
}

\begin{abstract}
In this paper we show how a simple modification of a well-known multiplicative process of firms' growth, taking into account common and idiosyncratic elements, allows to reconcile an old aggregate-sector puzzle (Quandt, 1966) on firms' size distribution reported in the literature.
\end{abstract}

Keywords: Firms' size, common components, Kesten's process. JEL classification codes: L11, O41.

\section{Introduction}

The heterogeneity of firms and the shape of their distribution is a phenomenon which has attracted considerable attention in the literature since the pioneering work of Gibrat, 1931, who found that the lognormal distribution characterized French industrial firms. Later on Simon 1955; Simon and Bonini, 1958; Ijiri and Simon, 1977; Steindl, 1965, argued that the firms' size distribution was right skew, leptokurtic and stable through time and countries.

Recently Axtell, 2001, and Gaffeo et al. 2003, have reported that the firms' size distribution of the most industrialised countries follows, approximatively, the Zipf distribution, i.e. a Pareto distribution with exponent close to one ${ }^{1}$.

Still 2 caveats are in order:

\footnotetext{
${ }^{1}$ This result is still under discussion. In Cabral-Mata (2003), using a Portuguese dataset
} 
- The Pareto distribution often holds above a given threshold (basically leaving outside the "small" firms). In empirical estimations the density of small firms are below the power law line. This means that they are many, but not so many as predicted by the theoretical distribution.

- This result is robust only at the aggregate level: if one analyzes sub-sectors (4-digits), the Pareto distribution is often rejected by statistical tests (Quandt, 1966).

To the best of our knowledge, there is not a standard explanation of the sectors puzzle. The only exception is Dosi (2007) in which aggregate "well-behaved" Pareto-type distributions may be caused by the aggregation of diverse manufacturing sectors, characterized by diverse regime of technological learning and market interactions, which do not display Paretian size distribution.

Generalizing this idea we want to explore the role of firms' comovements (caused by interaction, learning, imitation, etc.) in explaining the puzzle.

In doing so we generalize the Kesten's model (1973), that is essentially the Gibrat's random growth process with a lower bound, to take into account the presence of common components (e.g. common shocks) in the evolutions of firms' dynamics as an explanation of the two apparent distributions for small and big firms. Furthermore, we show that those common components, when dominant at the sector level, may destroy the Pareto features of the stochastic multiplicative processes. The higher the weight of the common factor the more the distribution of the firms deviates from a Pareto law, above all in the "small firms zone" of the support. There are many economic reasons to insert a common component in firm's dynamics. Firms evolve coordinated by the market.There are economic and sector specific trends and feedbacks from economy networks. When a system is interconnected (interaction among sectors is high), firm and sector specific shocks propagate through the system: this tantamount to say that they are equivalent to common factor shocks.

The importance of sector shocks in explaining aggregate fluctuations is discussed in Horvath (2003) while the empirical evidence at the firm level is analyzed in Baum, Mustafa and Neslihan (2003) where, using VARs and factor analytic methods, they show that both common and sector-specific innovations play a role in the observed comovement of sectoral business investment spending in the U.K. over

of manufacturing firms, it is argued that even though size distribution is non-lognormal in the aggregate it seems to converge to a lognormal for existing firms. They explain such phenomena with firm demography and financial contraints. 
the period 1980-2000. On average, 2 common factors explain $32 \%$ of the variation in firms' innovation series, with very weak effects in the mining and electronics sector, but very strong effects in the metals and utilities sectors.

In Shea (2002) local spillovers are important, explaining between $15 \%$ and $36 \%$ of manufacturing employment volatility.

Similar results are found in Alessi, Barigozzi and Capasso (2006) where, using the Generalized Dynamic Factor Model proposed by Forni et al. (2000), it is shown that 3 common factors may explain about $30 \%$ of the variance in output and investments firms' growth.

In the next section we show that, modifing the Kesten's process to take account of common components and calibrating it with the empirical weight that common factors have, according to the above literature, we are able to show that they be may an important source in the solution of the scaling puzzle.

Section 3 concludes.

\section{Multiplicative stochastic processes with common components}

Our starting point is the famous Gibrat's stochastic model leading to a lognormal distribution of firms' size and the Kesten process generating a power law distribution. The second one is defined by the following stochastic difference equation

$$
S_{i t}=\max \left[\left(1+g_{i t}\right) S_{i t-1}, \bar{S}\right]
$$

where $S_{i t}$ is a measure of firm- $i$ size (employees, output, capital, investment, etc), $\bar{S}$ is the minimum size, and $g_{i t}$ is the rate of growth from $t-1$ to $t$.

The process, provided the following condition

$$
E\left[\log \left(1+g_{i t}\right)\right]<0,
$$

is satisfied, generates a Pareto distribution with exponent near to unity ${ }^{2}$.

We use this process not because we think it is a realistic representation of firms' dynamics 3 , but because we want to show that simple multiplicative processes are consistent with empirical findings provided they include some economic considerations.

\footnotetext{
${ }^{2}$ Except for putting a positive probability mass to the case $S=\bar{S}$

${ }^{3}$ For example, the number of firms is held constant. There is no entry-exit dynamics.
} 
As said above, this simple model does not take care that there are common components in the evolution of the whole economic system and within sectors.

To explain the sector puzzle, we have to take into account comovements at the sector level, and so the following modification of the Kesten process can be written down

$$
S_{i j t}=\max \left[\left(1+g_{i j t}+\varepsilon_{j t}\right) S_{i j t-1}, \bar{S}\right]
$$

where $\varepsilon_{j t}$ is the sector- $j$ specific stochastic factor ${ }^{4}$.

The analysis of this modified integrate process, when the importance of the common component increase, led to the following

Proposition In the modified Kesten process, when sector specific components dominate, the Pareto distribution still hold at the aggregate level, but is destroyed at the sector one.

To prove the proposition first consider the case

$$
\begin{aligned}
& \operatorname{Var}\left(\varepsilon_{j t}\right)=\sigma_{\varepsilon}^{2} \rightarrow 0, \\
& \operatorname{Var}\left(g_{i j t}\right)=\sigma_{g}^{2}>0 .
\end{aligned}
$$

That is, the variance of the common factor goes to zero while the variance of the idiosyncratic shock is a positive parameter (i.e. there are no important common factors). In this case, the process reduces itself to the standard Kesten model.

On the contrary, when

$$
\begin{gathered}
\operatorname{Var}\left(\varepsilon_{j t}\right)=\sigma_{\varepsilon}^{2}>0, \\
\operatorname{Var}\left(g_{i j t}\right)=\sigma_{g}^{2} \rightarrow 0 .
\end{gathered}
$$

${ }^{4}$ The specification we choose is, in our opinion, the simplest setting to show the results. A more realistic representation will include connections and interactions between different sectors (input-output relations, demand-supply chains, etc.) and dynamical relationships. That is,

$$
S_{i j t}=\max \left[\left(1+g_{i j t}+a_{i j}(L) \epsilon_{t}+\sum_{j=1}^{J} b_{i j}(L) \varepsilon_{t}\right) S_{i j t-1}, \bar{S}\right]
$$

where $\sum b_{i j}(L) \varepsilon_{i j t}$ captures also the interaction structure between sectors and $\epsilon_{t}$ is an economy shock. The functions $a_{i j}(L)$ and $b_{i j}(L)$ are lag polynomials capturing the dynamical relationships. 
the process leads to degenerate distributions for each sectors. But with agents, in each sector, growing according to a standard Kesten process generating a Pareto distribution for the whole economy provided that condition (1) is satisfied ${ }^{5} \square$.

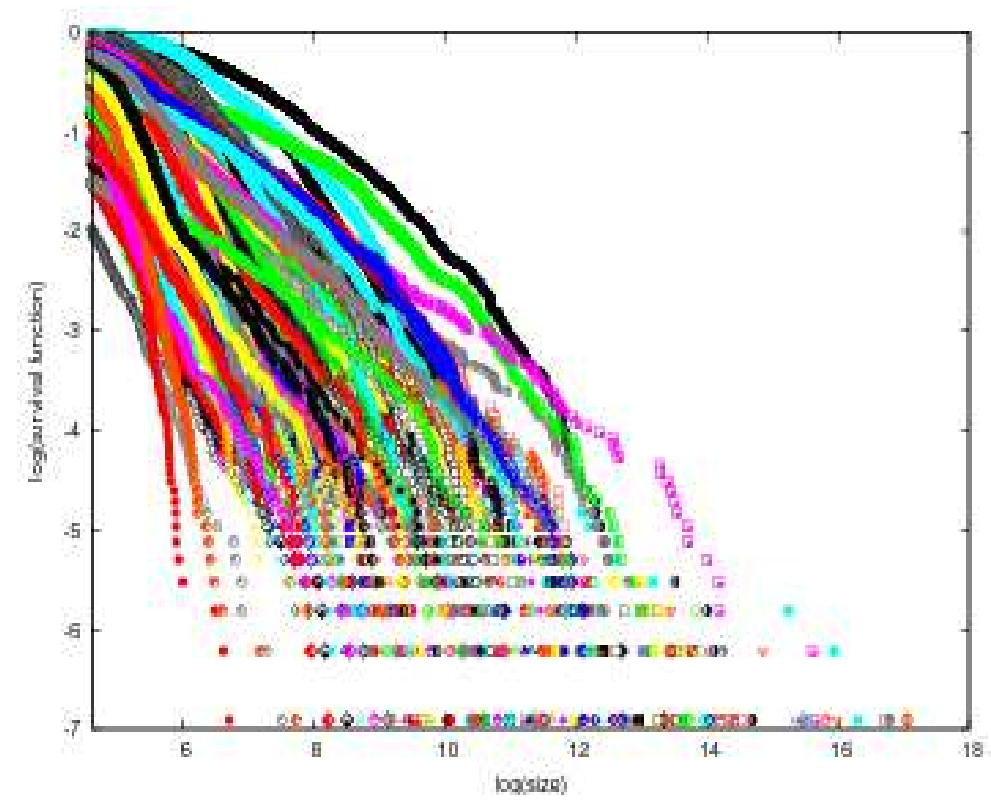

Figure 1: log-log of firms' size distribution in every sector for the modified Kesten's process. In the abscissa there is the log of firm's size and in the ordinate the log of the survival function (i.e., one minus the cumulative distribution function.)

To show what happens in non degenerate situations, we perform a 1000 periods simulation of the modified Kesten's process with 100 sectors and 1000 firms in each sector 6 . The two factors $g_{i j t}$ and $\varepsilon_{j t}$ are both drawn from a normal distribution. The variance of $g_{i j t}+\varepsilon_{j t}$ is 1 and the weight of $\varepsilon_{j t}$ is $30 \%$. In other terms, as said above, the multiplicative process at the firm's level follows the Kesten assumptions except for

\footnotetext{
${ }^{5}$ In other terms, the process reduces to a Kesten one in which the role of firms is played by sectors.

${ }^{6}$ The simulation was implemented in GNU Octave; an high-level language, mostly compatible with Matlab, primarily intended for numerical computations (see Eaton, 2002).
} 


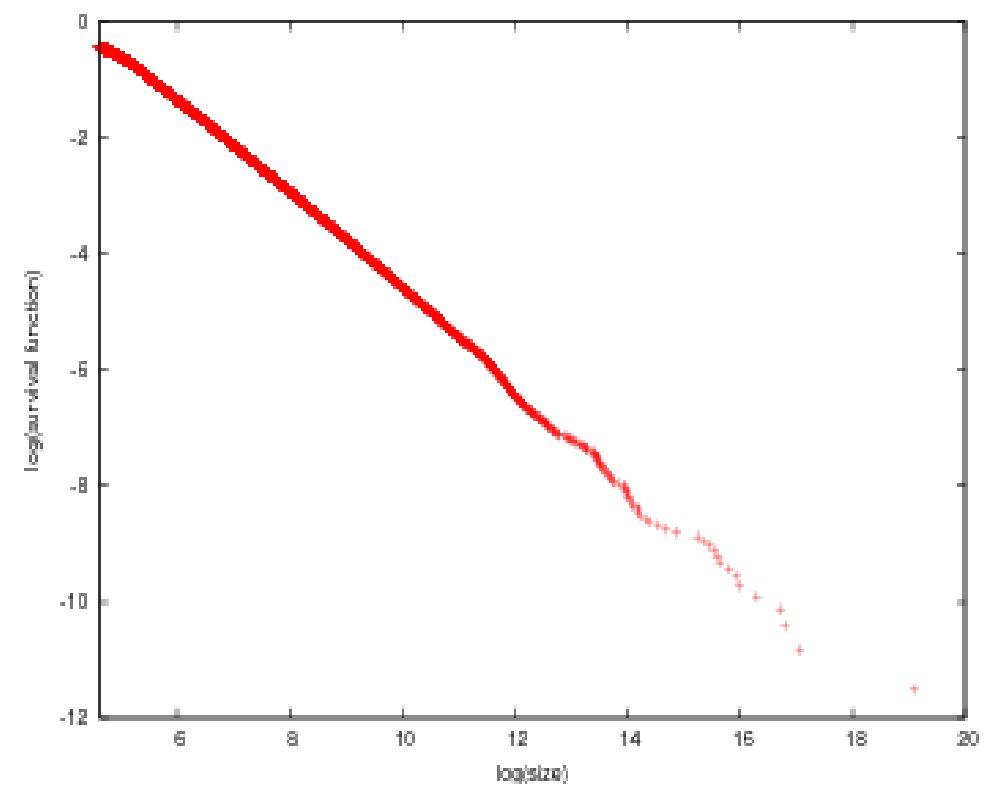

Figure 2: Pareto shape for the aggregate simulated economy.

the presence of a sector specific common components at time $t$, with a weight comparable to what found in the empirical literature.

Initial size is set equal to 1000 and the minimum size, $\bar{S}$, equal to 100.

Figures 2 and 3 show that the presence of the common components destroys the Pareto distribution in many sectors (in figure 2 is drawn the log-log of firms' size distribution in every sector; the KolmogorovSmirnov test rejects the Pareto distribution in 58 of the 100 sectors) but not in the whole economy (figure 3).

From the Gibrat's analysis we know that multiplicative processes generate a lot of heterogeneity that end up with log-normal or, as discovered later, Pareto type distributions. The presence of common components, obviously, delay or destroy such heterogeneity generating process at the sector level. In particular, since small firms are those that, by chance, have received small idiosyncratic shocks, their evolution is ruled by common components. The explanation of the apparent paradox - in this simple theoretical model - regarding the distribution at the aggregate and sector level, is that even though the common components reduce the fatness in the tails of sector distributions, the process generates high level of heterogeneity across sectors. Aggregating them up will reconstruct a power law behavior for the whole economy. 


\section{Conclusions}

The literature on firms' size distribution, although old and authoritative, has not reached a clear conclusion, because of the different distributions within/across different sectors. In this paper we have shown how a simple modification of the Kesten model, taking into account common and idiosyncratic elements allows to reconcile the puzzles on firms' distribution reported in the literature. We believe, the presence of common components in the evolutions of firms may be considered as an important factor in determining the different distributions holding for the small and the large firms. But there may be others relevant explanations. What this paper shows is the consistency of a plethora of different distributions, at the sector level, when the aggregate economy exhibits scaling invariance features.

We have also shown that, when the common component is the dominant factor at the sector level, it can determine the breakdown of the Pareto distribution itself.

\section{References}

[1] Alessi L., Barigozzi M. and Capasso M. (2006), A dynamic factor analysis of business cycle on firm-level data, LEM Working Paper $2006 / 27$.

[2] Axtell, R. (2001), Zipf Distribution of U.S. firm sizes. Science 293, 1818-1820.

[3] Axtell, R. (2006), Firm Sizes: Facts, Formulae and Fantasies. CSED Working Paper No.44.

[4] Baum C., Mustafa C. and Neslihan O. (2003), Sectoral fluctuations in U.K. firms' investment expenditures. Economics Bulletin, Vol. 5, No. 13 pp. 1 ?10.

[5] Cabral L.M.B., Mata J. (2003), On the Evolution of the Firm Size Distribution: Facts and Theory, American Economic Review 93, 1075-1090.

[6] Dosi, G. (2007), Statistical regularities in the evolution of industries: a guide through some evidence and challenges fotf the theory. In Malerba, F., Brusoni S. (eds.), Perspective and Innovation, Cambridge University Press.

[7] Eaton J.W. (2002), GNU Octave Manual, Network Theory Limited. URL: http://www .octave.org 
[8] Forni M., Hallin M., Lippi M. and Reichlin L. (2000), The generalized dynamic factor model: identification and estimation, The Review of Economics and Statistics, 82:540554.

[9] Gaffeo, E., Gallegati, M., Palestrini, A. (2003), On the size distribution of firms. Additional evidence from the G7 countries. Physica A 324, 117-123.

[10] Gibrat, R. (1931), Les inégalités économiques; applications: aux inégalités des richesses, à la concentration des entreprises, aux populations des villes, aux statistiques des familles, etc., d'une loi nouvelle, la loi de l'effet proportionnel. Paris: Librairie du Recueil Sirey.

[11] Horvath M. (2000), Sectoral shocks and aggregate fluctuations, Journal of Monetary Economics, Volume 45, Issue 1, pp. 69-106

[12] Ijiri, Y., Simon, H.A., (1977), Skew Distributions and the Sizes of Business Firms, North Holland, Amsterdam.

[13] Kesten, H. 1973. Random Difference Equations and Renewal Theory for Products of Random Matrices. Acta Mathematica, CXXXI: 207-248.

[14] Pareto V. (1897), Cours d'Economie Politique, Vol. 2, Paris, F. Pichou.

[15] Quandt, R. (1966), On the Size Distribution of Firms. American Economic Review 56: 416-432.

[16] Shea J. (2002), Complementarities and Comovements, Journal of Money, Credit and Banking, 34:2., pp. 412-433.

[17] Simon, H. A. (1955). On a Class of Skew Distribution Functions. Biometrika 42: 425-440.

[18] Simon, H. A. (1957). Models of Man: Social and Rational. New York, N.Y., John Wiley \& Sons, Inc.

[19] Simon, H. A. and C. Bonini (1958). The Size Distribution of Business Firms. American Economic Review 48(4): 607-617.

[20] Steindl, J. (1965). Random Processes and the Growth of Firms. New York, N.Y., Hafner Publishing Company. 